\title{
FINITE LATTICES AND JORDAN-HÖLDER SETS ${ }^{1}$ )
}

\author{
RICHARD P. STANLEY
}

\section{Introduction}

In this paper we extend some aspects of the theory of 'supersolvable lattices' [3] to a more general class of finite lattices which includes the upper-semimodular lattices. In particular, all conjectures made in [3] concerning upper-semimodular lattices will be proved. For instance, we will prove that if $L$ is finite upper-semimodular and if $L^{\prime}$ denotes $L$ with any set of 'levels' removed, then the Möbius function of $L$ ' alternates in sign. Familiarity with [3] will be helpful but not essential for the understanding of the results of this paper. However, many of the proofs are identical to the proofs in [3] (once the machinery has been suitably generalized) and will be omitted.

\section{Admissible labelings}

Let $L$ be a finite lattice with bottom $\hat{0}$ and top $\hat{1}$, such that every maximal chain of $L$ has the same length $n$. Hence $L$ has a rank function $\varrho$ satisfying $\varrho(\hat{0})=0, \varrho(\hat{1})=n$, and $\varrho(y)=1+\varrho(x)$ whenever $y$ covers $x$ in $L$. We call $L$ a graded lattice.

Let $I$ denote the set of join-irreducible elements of $L$. A labeling $\omega$ of $L$ is any map $\omega: I \rightarrow \mathbf{P}$, where $\mathbf{P}$ denotes the positive integers. A labeling $\omega$ is said to be natural if $z, z^{\prime} \in I$ and $z \leq z^{\prime}$ implies $\omega(z) \leq \omega\left(z^{\prime}\right)$. If $x<y$ in $L$ and $\omega$ is a fixed labeling of $L$, define

$$
\gamma(x, y)=\min \{\omega(z) \mid z \in I, x<x \vee z \leq y\} .
$$

Thus, $\gamma(x, y)$ is the least label of a join-irreducible which is less than or equal to $y$ but not less than or equal to $x$. Note that $\gamma(x, y)$ is always defined since $y$ is a join of join-irreducibles. We are now able to make the key definition of this paper. A labeling $\omega$ is said to be admissible if whenever $x<y$ in $L$, there is a unique unrefinable chain $x=x_{0}<x_{1}<\cdots<x_{m}=y$ between $x$ and $y$ (so $m=\varrho(y)-\varrho(x)$ ) such that

$$
\gamma\left(x_{0}, x_{1}\right) \leq \gamma\left(x_{1}, x_{2}\right) \leq \cdots \leq \gamma\left(x_{m-1}, x_{m}\right) .
$$

We then call the pair $(L, \omega)$ an admissible lattice. Our motivation for this definition is that admissibility seems to be the weakest condition for which Theorem 3.1 holds.

1) The research was supported by a Miller Research Fellowship at the University of California at Berkeley. 
The idea for this definition came from [3, Cor. 1.3] and its relation to [3, Thm. 2.1]. Our present Theorem 3.1 is a generalization of [3, Thm. 2.1].

We first note a simple property of admissible labelings.

2.1. PROPOSITION. Let $\omega$ be an admissible labeling of a finite graded lattice $L$. Then $\omega$ is natural.

Proof. Suppose $z, z^{\prime} \in I$ with $z<z^{\prime}$ and $\omega(z)>\omega\left(z^{\prime}\right)$. Since $z$ is join-irreducible, it covers a unique element $x$. Similarly $z^{\prime}$ covers a unique element $y$. Hence any unrefinable chain between $x$ and $z^{\prime}$ has the form $x<z=y_{0}<y_{1}<\cdots<y_{m}=y<z^{\prime}$ (possibly $m=0$ so $z=y$ ). Since $z$ is join-irreducible, it follows from the definition of $y$ that $\gamma(x, z)=\omega(z)$. Similarly $\gamma\left(y, z^{\prime}\right)=\omega\left(z^{\prime}\right)$. Since $\omega(z)>\omega\left(z^{\prime}\right), \omega$ cannot be admissible.

We know of two main classes of admissible lattices. The first class is given by the next proposition.

First recall that a lattice $L$ of finite length is said to upper-semimodular if it is a graded lattice whose rank function $\varrho$ satisfies $\varrho(x)+\varrho(y) \geq \varrho(x \vee y)+\varrho(x \wedge y)$ for all $x, y \in L$. Equivalently, $L$ is upper-semimodular if whenever $x$ covers $y$, then $x \vee z$ covers or equals $y \vee z$, for all $x, y, z \in L$.

2.2. PROPOSITION. Let $L$ be a finite upper-semimodular lattice and $\omega$ a natural labeling of $L$ such that whenever $z$ and $z^{\prime}$ are incomparable join-irreducibles then $\omega(z) \neq \omega\left(z^{\prime}\right)$. (Such a labeling of $L$ is clearly possible; in fact, an injective natural labeling can always be found.) Then $\omega$ is admissible.

To prove this result, we first need a lemma.

2.3. LEMMA. Let $(L, \omega)$ satisfy the hypotheses of Proposition 2.2, and let $x<y$ in $L$. Let $z$ be a minimal element of the set $J$ of all join-irreducibles $z^{\prime}$ of $L$ satisfying $\omega\left(z^{\prime}\right)=\gamma(x, y)$ and $x<x \vee z^{\prime} \leq y .(J$ is not empty by definition of $\gamma(x, y)$.) Then $x \vee z$ covers $x$.

Proof. Let $I$ denote as before the set of join-irreducibles of $L$. Let $I^{\prime} \subseteq I$ be the set of all $z^{\prime} \in I$ satisfying $z^{\prime}<z$. Let $z^{\prime} \in I^{\prime}$. Since $z^{\prime}<z, x \leq x \vee z^{\prime} \leq y$. Since $\omega$ is natural, $\omega\left(z^{\prime}\right) \leq \omega(z)$. If $\omega\left(z^{\prime}\right)<\omega(z)$, then by definition of $\gamma(x, y)$ we cannot have $x<x \vee z^{\prime} \leq y$, so $x=x \vee z^{\prime}$. On the other hand, if $\omega\left(z^{\prime}\right)=\omega(z)$, then by hypothesis we cannot have $x<x \vee z^{\prime} \leq y$, so once again $x=x \vee z^{\prime}$. Thus $x=x \vee z^{\prime}$ for all $z^{\prime} \in I^{\prime}$. Let $w=\vee_{z^{\prime} \in I^{\prime}} z^{\prime}$. Since $z$ is join-irreducible, $w<z$. Since $x=x \vee z^{\prime}$ for all $z^{\prime} \in I^{\prime}$, we have $x \vee w=x$.

Now if $z$ doesn't cover $w$, then $w<w^{\prime}<z$ for some $w^{\prime} \in L$. But then there is a new join-irreducible $v<z$ such that $w<w \vee v \leq w^{\prime}$, contradicting the definition of $w$. Hence $z$ covers $w$. But by upper-semimodularity, if $z$ covers $w$, then $x \vee z$ covers or equals $x \vee w=x$. By assumption, $x<x \vee z$, so $x \vee z$ covers $x$.

Proof of Proposition 2.2. Let $x<y$ in $L$, and let $m=\varrho(y)-\varrho(x)$. We first show the existence of an unrefinable chain $x=x_{0}<x_{1}<\cdots<x_{m}=y$ between $x$ and $y$ satis- 
fying (1). Let $z_{1}$ be a minimal element of the set $J_{1}$ of join-irreducibles $z$ satisfying $\omega(z)=\gamma\left(x_{0}, y\right)$ and $x<x \vee z \leq y$. Let $x_{1}=x_{0} \vee z_{1}$. By Lemma 2.3, $x_{1}$ covers $x_{0}$, while by definition $x_{1} \leq y$.

If $m=1$, we are done, so assume $m \geq 2$. Let $z_{2}$ be a minimal element of the set $J_{2}$ of join-irreducibles $z$ satisfying $\omega(z)=\gamma\left(x_{1}, y\right)$ and $x_{1}<x_{1} \vee z \leq y$. Let $x_{2}=x_{1} \vee z_{2}$. Once again by Lemma $2.3 x_{2}$ covers $x_{1}$, while again by definition $x_{2} \leq y$. Now by definition of $\gamma\left(x_{0}, y\right)$ we have $\omega\left(z_{1}\right)=\gamma\left(x_{0}, y\right) \leq \omega\left(z_{2}\right)=\gamma\left(x_{1}, y\right)$. Continuing in this way, after $m$ steps we get an unrefinable chain $x=x_{0}<x_{1}<\cdots<x_{m}=y$ satisfying $\gamma\left(x_{0}, y\right) \leq \gamma\left(x_{1}, y\right) \leq \cdots \leq \gamma\left(x_{m-1}, y\right)$. But clearly by definition of $\gamma$ and the $x_{i}$ 's, $\gamma\left(x_{i}, y\right)=\gamma\left(x_{i}, x_{i+1}\right)$. Hence we have constructed a chain $C$ satisfying (1).

It remains to show the uniqueness of $C$. We shall prove the following two results:

(i) If $x^{\prime} \in L$ is such that $x^{\prime}$ covers $x, x^{\prime} \leq y$, and $\gamma\left(x, x^{\prime}\right)=\gamma(x, y)$, then $x^{\prime}=x_{1}$;

(ii) If $x=x_{0}^{\prime}<x_{1}^{\prime}<\cdots<x_{m}^{\prime}=y$ is any unrefinable chain satisfying (1), then $\gamma\left(x_{1}^{\prime}, x\right)=\gamma(x, y)$.

Thus (i) and (ii) imply that $x_{1}^{\prime}$ is uniquely determined, viz., $x_{1}^{\prime}=x_{1}$ (where $x_{1}$ $=x_{0} \vee z_{1}$ as defined above). Hence the proof of the proposition follows by induction on $m$.

Proof of (i). Suppose $x^{\prime \prime} \neq x^{\prime}$ also is such that $x^{\prime \prime}$ covers $x, x^{\prime \prime} \leq y$, and $\gamma\left(x, x^{\prime \prime}\right)$ $=\gamma(x, y)$. Thus there exist $z^{\prime}, z^{\prime \prime} \in I$ such that $\omega\left(z^{\prime}\right)=\omega\left(z^{\prime \prime}\right)=\gamma(x, y), x \vee z^{\prime}=x^{\prime}$, $x \vee z^{\prime \prime}=x^{\prime \prime}$. Since $x^{\prime}$ and $x^{\prime \prime}$ both cover $x$, they are incomparable. Hence $z^{\prime}$ and $z^{\prime \prime}$ are incomparable. Thus by hypothesis $\omega\left(z^{\prime}\right) \neq \omega\left(z^{\prime \prime}\right)$, a contradiction. Hence $x^{\prime \prime}$ cannot exist.

Proof of (ii). Let $x=x_{0}^{\prime}<x_{1}^{\prime}<\cdots<x_{m}^{\prime}=y$ be an unrefinable chain satisfying (1). Hence $\gamma\left(x, x_{1}^{\prime}\right) \geq \gamma(x, y)$. Suppose $\gamma\left(x, x_{1}^{\prime}\right)>\gamma(x, y)$. Let $z \in I$ satisfy $\omega(z)=\gamma(x, y)$ and $x<x \vee z \leq y$. Let $i$ be the least positive integer for which $x \vee z \leq x_{i}^{\prime}$. (Clearly $i$ exists since $x \vee z \leq x_{m}^{\prime}$.) Then $x_{i-1}^{\prime} \vee z=x_{i}^{\prime}$, so $\gamma\left(x_{i-1}^{\prime}, x_{i}^{\prime}\right)=\gamma(x, y)<\gamma\left(x, x_{1}^{\prime}\right)$. Thus (1) cannot hold.

The second main class of admissible lattices are the supersolvable lattices [3]. If $L$ is a finite lattice and $\Delta$ a maximal chain of $L$, we call the pair $(L, \Delta)$ a supersolvable lattice (or $S S$-lattice) if the sublattice of $L$ generated by $\Delta$ and any chain in $L$ is distributive. It is easily seen that if $(L, \Delta)$ is an $S S$-lattice, then $L$ is graded (cf. $[3, \S 1]$ ).

2.4. PROPOSITION. Let $(L, \Delta)$ be an SS-lattice with $\Delta$ given by $\hat{0}=x_{0}<x_{1}<$ $<\cdots<x_{n}=\hat{1}$. Define a labeling $\omega: I \rightarrow \mathbf{P}$ by letting $\omega(z)$ be the least positive integer $t$ for which $z \leq x_{t}$. Then $\omega$ is admissible

Proof. Recall that an interval $[u, v]$ of a lattice is prime if it contains exactly two elements, i.e., if $v$ covers $u$. In a distributive lattice $D$, two prime intervals $[x, y]$ and $[u, v]$ are said to be projective if there is a unique join-irreducible $z$ such that $y=x \vee z$ and $v=u \vee z$. This is easily seen to be equivalent to the usual definition of projectivity (e.g., $[1$, p. 14]) if one thinks of $D$ as being coordinatized by a ring of sets. 
If $y$ covers $x$ in $L$, then it is easily seen [3, p. 198] that there is a unique positive integer $t$, which we denote by $\gamma^{\prime}(x, y)$, for which the prime intervals $[x, y]$ and $\left[x_{t-1}, x_{t}\right]$ are projective in the distributive lattice $D_{x y}$ generated by $\Delta$ and $\{x, y\}$. In [3, Cor. 1.3], it was shown that for any $x^{\prime}<y^{\prime}$ in $L$, there is a unique unrefinable chain $x^{\prime}=x_{0}^{\prime}<x_{1}^{\prime}<\cdots<x_{m}^{\prime}=y$ between $x^{\prime}$ and $y^{\prime}$ such that

$$
\gamma^{\prime}\left(x_{0}^{\prime}, x_{1}^{\prime}\right)<\gamma^{\prime}\left(x_{1}^{\prime}, x_{2}^{\prime}\right)<\cdots<\gamma^{\prime}\left(x_{m-1}^{\prime}, x_{m}^{\prime}\right) \text {. }
$$

Hence it suffices to prove that $\gamma(x, y)=\gamma^{\prime}(x, y)$ whenever $y$ covers $x$.

We shall need the following elementary facts concerning projectivity in a finite distributive lattice $D$. The proofs are immediate from the above definition of projectivity.

(a) The prime intervals $[x, y]$ and $[u, v]$ are projective in $D$ if and only if $x=(x \vee u) \wedge y$ and $y=(x \vee v) \wedge y$.

(b) If $[x, y]$ and $[u, v]$ are projective prime intervals in $D$, then $y \$ u$.

(c) Suppose $[w, z]$ is a prime interval in $D$ and $z$ is join-irreducible. If $y$ covers $x$ in $D$ and $z \leq y, z \$ x$, then $[w, z]$ and $[x, y]$ are projective.

We proceed to prove that if $y$ covers $x$ in $L$, then $\gamma(x, y)=\gamma^{\prime}(x, y)$. By definition of $\gamma(x, y)$, there is a join-irreducible $z$ satisfying $x \vee z=y$ and $\omega(z)=\gamma(x, y)$. Let $w$ be the unique element of $L$ covered by $z$, and set $s=\omega(z)$. By (c), $[w, z]$ and $\left[x_{s-1}, x_{s}\right]$ are projective in the distributive lattice $D_{w z}$ generated by $\Delta$ and $\{w, z\}$, so $\gamma^{\prime}(w, z)=s$. If $z^{\prime}$ is a join-irreducible of $L$ such that $z^{\prime}<z$, then it follows from (b) (taking $D$ to be generated by $\Delta$ and $\left.\left\{z, z^{\prime}\right\}\right)$ that $\omega\left(z^{\prime}\right) \neq \omega(z)$. Since $\omega\left(z^{\prime}\right) \leq \omega(z)$, thus $\omega\left(z^{\prime}\right)<\omega(z)$.

We claim that $w \leq x$. It suffices to prove $z^{\prime} \leq x$ for all join-irreducibles $z^{\prime} \leq w$. If $z^{\prime}$ is such a join-irreducible, then by the above $\omega\left(z^{\prime}\right)<\omega(z)$. Hence by the definition of $z, x \vee z^{\prime}=y$. But $x \vee z^{\prime} \leq y$ since $z \leq y$. Since $y$ covers $x$, we must have $z^{\prime} \leq x$. Hence $w \leq x$.

We need to show $\omega(z)=t$, i.e., $s=t$. By (a) and (c) this is equivalent to $w=\left(w \vee x_{t-1}\right)$ $\wedge z$ and $z=\left(w \vee x_{t}\right) \wedge z$. Since $\gamma^{\prime}(x, y)=t$, we know by (a) that

$$
\begin{aligned}
& x=\left(x \vee x_{t-1}\right) \wedge y \\
& y=\left(x \vee x_{t}\right) \wedge y .
\end{aligned}
$$

Since $w \leq x, z \$ x, z \leq y$, and $z$ covers $w$, from (2) we get $w=x \wedge z=\left(x \vee x_{t-1}\right) \wedge z$. Thus since $w \leq x$ and $w \leq z, w \leq\left(w \vee x_{t-1}\right) \wedge z \leq\left(x \vee x_{t-1}\right) \wedge z=w$ so $w=\left(w \vee x_{t-1}\right) \wedge z$ as desired. To prove the other equality $z=\left(w \vee x_{t}\right) \wedge z$, we need to show $w \vee x_{t} \geq z$. Since $w$ is the only element which $z$ covers, this is equivalent to $x_{t} \$ w$. But if $x_{t} \leq w$, then $x_{t} \leq x$ since $w \leq x$. From (3) this would imply $y=x \wedge y=x$, a contradiction.

It follows from Proposition 2.4 that the theory of $S S$-lattices, as developed in [3], is a special case of the theory of admissible lattices. A large class of examples of $S S$ lattices, some of which are not semimodular, is given in $[3, \S 2]$. 


\section{Jordan-Hölder sequences}

Let $(L, \omega)$ be an admissible finite graded lattice. Let $x \leq y$ in $L$, and suppose $K$ is an unrefinable chain in $L$ between $x$ and $y$ given by $x=x_{0}<x_{1}<\cdots<x_{m}=y$. Define the Jordan-Hölder sequence (or $J$-H sequence) associated with $K$ to be the sequence $a_{1}, a_{2}, \ldots, a_{m}$ of positive integers given by $a_{i}=\gamma\left(x_{i-1}, x_{i}\right)$. We shall denote this sequence by $\pi_{K}$ and shall write

$$
\pi_{K}=\left(a_{1}, a_{2}, \ldots, a_{m}\right)
$$

In [3] $\pi_{K}$ was called a ' $J-H$ permutation' but here repetitions among the $a_{i}$ are possible.

Now define the Jordan-Hölder set (or $J-H$ set) $\mathscr{J}_{x y}(L, \omega)$ of $(L, \omega ; x, y)$ (denoted $\mathscr{J}_{x y}$ for short) to be the set of all $J-H$ sequences $\pi_{K}$, including repetitions, as $K$ ranges over all unrefinable chains between $x$ and $y$. It follows from the definition of an admissible labeling that there is a unique element $\pi_{K}=\left(a_{1}, \ldots, a_{m}\right)$ of $\mathscr{J}_{x y}$ satisfying $a_{1} \leq a_{2} \leq \cdots \leq a_{m}$. If $x=\hat{0}$ and $y=\hat{1}$, we denote $\mathscr{J}_{x y}(L, \omega)$ simply by $\mathscr{J}(L, \omega)$ or just $\mathscr{J}$, and call it the $J-H$ set of $(L, \omega)$.

If $k \in \mathbf{P}$, let $\mathbf{k}$ denote the set $\{1,2, \ldots, k\}$. We also write $S=\left\{m_{1}, m_{2}, \ldots, m_{s}\right\}<$ to signify that $S=\left\{m_{1}, m_{2}, \ldots, m_{s}\right\}$ and $m_{1}<m_{2}<\cdots<m_{s}$. Suppose $L$ is a finite graded lattice and $[x, y]$ is an interval of $L$ of length (rank) $m$, i.e., $\varrho(y)-\varrho(x)=m$. If $\left\{m_{1}, \ldots, m_{s}\right\}_{<}=S \subseteq \mathbf{m}-1$, define $\alpha_{x y}(S)$ to be the number of chains

$$
x<y_{1}<\cdots<y_{s}<y
$$

in $L$ satisfying $\varrho\left(y_{i}\right)-\varrho(x)=m_{i}, i=1,2, \ldots, s$. Thus if $S=\{k\}$, then $\alpha_{x y}(S)$ is the number of elements $z$ of $[x, y]$ of rank $k$ in $[x, y]$ (i.e., $\varrho(z)-\varrho(x)=k$ ). Moreover, $\alpha_{x y}(\phi)=1$ and $\alpha_{x y}(\mathbf{m}-1)$ is the total number of unrefinable chains in $L$ between $x$ and $y$. Now define for $S \subseteq \mathbf{m}-\mathbf{1}$,

$$
\beta_{x y}(S)=\sum_{T \leq S}(-1)^{|S-T|} \alpha_{x y}(T),
$$

so by the Principle of Inclusion-Exclusion [2],

$$
\alpha_{x y}(S)=\sum_{T \subseteq S} \beta_{x y}(T)
$$

As mentioned in [3, p. 198], if $L_{x y}(S)$ denotes the partially ordered set of all $z \in L$ satisfying either (a) $z=x$; (b) $z=y$; or (c) $x<z<y$ and $\varrho(z)-\varrho(x) \in S$, then

$$
\mu_{S}(x, y)=(-1)^{s+1} \beta_{x y}(S) \text {, }
$$

where $\mu_{S}$ is the Möbius function of $L_{x y}(S)$ and $|S|=s$. For this reason we call the function $\beta_{x y}(\cdot)$ the rank-selected Möbius invariant of the interval $[x, y]$. 
If $\pi=\left(a_{1}, a_{2}, \ldots, a_{m}\right)$ is a finite sequence of integers, then a pair $a_{j}>a_{j+1}$ is called a descent of $\pi$, and the set

$$
D(\pi)=\left\{j: a_{j}>a_{j+1}\right\}
$$

is called the descent set of $\pi$. We can now state the fundamental combinatorial property of $J-H$ sets. This result is a direct generalization of [3, Thm. 1.2]. The proof is identical to the proof of [3, Thm. 1.2], except that here the definition of an admissible lattice plays the role of Lemma 3.1 of [3]. Thus no condition is needed about distributive sublattices of $L$.

3.1. THEOREM. Let $(L, \omega)$ be an admissible lattice, and let $[x, y]$ be an interval of $L$ of length $m$. If $S \subseteq \mathbf{m}-1$, then the number of sequences $\pi$ in the J-H set $\mathscr{J}_{x y}(L, \omega)$ with descent set $D(\pi)=S$ is equal to $\beta_{x y}(S)$. (The reader is reminded that $\mathscr{J}_{x y}(L, \omega)$ contains one sequence $\pi$ for each maximal chain of $[x, y]$, so that repeated sequences are taken into account.)

3.2. COROLLARY. Let $(L, \omega)$ be an admissible lattice. If $[x, y]$ is an interval of $L$ of length $m$ and if $S \subseteq \mathbf{m}-\mathbf{1}$, then $\beta_{x y}(S) \geq 0$.

In view of (4), Corollary 3.2 may be restated as follows:

3.2'. COROLLARY. Let $(L, \omega)$ be an admissible lattice of length $n$, and let $S \subseteq \mathrm{n}-1$. Then the Möbius function $\mu_{S}$ of the rank-selected partially ordered set $L(S)$ alternates in sign; i.e., if $[x, y]$ is an interval in $L(S)$ of length $k$, then

$$
(-1)^{k} \mu_{S}(x, y) \geq 0 \text {. }
$$

Since by Proposition 2.2 every finite upper-semimodular lattice has an admissible labeling, Corollary $3.2^{\prime}$ applies to all such lattices, and in particular, to finite geometric lattices.

3.3. COROLLARY. Let $(L, \omega)$ be an admissible lattice and $[x, y]$ an interval of $L$ of length $m$. Let $\mu$ denote the Möbius function of $L$. Then $(-1)^{m} \mu(x, y)$ is equal to the number of unrefinable chains $x=x_{0}<x_{1}<\cdots<x_{m}=y$ between $x$ and $y$ satisfying

$$
\gamma\left(x_{0}, x_{1}\right)>\gamma\left(x_{1}, x_{2}\right)>\cdots>\gamma\left(x_{m-1}, x_{m}\right) \text {. }
$$

Proof. Let $S=\mathbf{m}-\mathbf{1}$ in Theorem 3.1, and use (4).

\section{Applications}

We shall state those results in [3] proved for SS-lattices which remain true for admissible lattices. The proofs are exactly the same as in the $S S$-case once suitable 
analogues are given for two concepts in [3]. First, the role of the induced $M$-chain $\Delta_{x y}$, between $x$ and $y$ ' is replaced by the unique unrefinable chain $x=x_{0}<x_{1}<\cdots<x_{m}=y$ between $x$ and $y$ satisfying $\gamma\left(x_{0}, x_{1}\right) \leq \gamma\left(x_{1}, x_{2}\right) \leq \cdots \leq \gamma\left(x_{m-1}, x_{m}\right)$. Secondly, we need a replacement for statement (A) in the proof of Theorem 5.2 of [3]. Although a direct analogue of $(A)$ can be given, it is simpler to use the following fact:

4.1. LEMMA. If $[x, y]$ is an interval of an upper-semimodular admissible lattice $(L, \omega)$ such that $y$ is the join of atoms of $[x, y]$, then there is an unrefinable chain $x=x_{0}<x_{1}<\cdots<x_{m}=y$ between $x$ and $y$ such that $\gamma\left(x_{0}, x_{1}\right)>\gamma\left(x_{1}, x_{2}\right)>\cdots>$ $\gamma\left(x_{m-1}, x_{m}\right)$.

Proof. Recall that a geometric lattice is an upper-semimodular lattice whose joinirreducibles are its atoms. If $L^{\prime}$ denotes the partially ordered set of all elements of $[x, y]$ which are a join of atoms of $[x, y]$ (including $x$ as the void join), then $L^{\prime}$ has the structure of a geometric lattice (though $L^{\prime}$ is not necessarily a sublattice of $L$ ). If $\mu$ denotes the Möbius function of $L$ and $\mu^{\prime}$ that of $L^{\prime}$, then from [2, Cor. on p. 349] we conclude $\mu(x, y)=\mu^{\prime}(x, y)$. Hence by $[2, \S 7$, Thm. 4], $\mu(x, y) \neq 0$. The desired result now follows from Corollary 3.3 .

The reader can now verify that the proofs of the following results are the same as the analogous results for $S S$-lattices given in [3].

4.2. PROPOSITION. (Generalizes [3, Prop. 3.3]). Let $(L, \omega)$ be an admissible lattice, and let $[x, y]$ be an interval of $L$ length $m$. Let $S \subseteq \mathbf{m}-1$. If $\beta_{x y}(S)>0$ and $T \subseteq S$, then $\beta_{x y}(T)>0$.

Suppose $L$ is a finite geometric lattice. Then $L$ is upper-semimodular, so by Proposition 2.2 $L$ possesses an admissible labeling. Moreover, every interval of $L$ is a geometric lattice, and the Möbius function of $L$ is never 0 . It follows from (4) and Proposition 4.2 that Corollary 3.2' can be strengthened in the case of geometric lattices as follows:

4.3. COROLLARY. Let $L$ be a finite geometric lattice of rank $n$, and let $S \subseteq \mathbf{n}-1$. Then the Mobius function $\mu_{S}$ of the rank-selected partially ordered set $L(S)$ strictly alternates in sign; i.e., if $[x, y]$ is an interval in $L(S)$ of length $k$, then

$$
(-1)^{k} \mu_{S}(x, y)>0 \text {. }
$$

For some related properties of geometric lattices, see the next section.

Recall $[3, \S 5]$ that a Loewy chain between $x$ and $y$ in a lattice $L$ of finite length is a chain $x=x_{0}<x_{1}<\cdots<x_{r}=y$ such that each $x_{i}, i \in \mathbf{r}$, is the join of the atoms of the interval $\left[x_{i-1}, x_{i}\right]$.

4.4. PROPOSITION. (Generalizes [3, Lemma 5.1]). Let $(L, \omega)$ be an admissible 
lattice with $[x, y]$ an interval of length $m$. Let $K$ be an unrefinable chain in $L$ between $x$ and $y$ :

$$
K: x=y_{0}<y_{1}<\cdots<y_{m}=y .
$$

Let $0<m_{1}<m_{2}<\cdots<m_{r}=m$. Then the subchain

$$
x=y_{0}<y_{m_{1}}<y_{m_{2}}<\cdots<y_{m_{r}}=y
$$

of $K$ is a Loewy chain between $x$ and $y$ if

$$
\mathbf{m}-\mathbf{1}-D\left(\pi_{K}\right) \subseteq\left\{m_{1}, m_{2}, \ldots, m_{r-1}\right\} .
$$

4.5. THEOREM. (Generalizes [3, Thm. 5.2]). Let L be a finite upper-semimodular lattice with $[x, y]$ an interial of $L$ of length $m$. Let $S=\left\{m_{1}, m_{2}, \ldots, m_{s}\right\}<\subseteq \mathrm{m}-1$. There exists a chain $C$.

$$
C: x=y_{0}<y_{1}<\cdots<y_{s}<y_{s+1}=y
$$

satisfying the two conditions

(i) $\varrho\left(y_{i}\right)-\varrho(x)=m_{i}, 1 \leq i \leq s$ (where $\varrho$ as usual is the rank function of $L$ );

(ii) $C$ is a Loewy chain between $x$ and $y$, if and only if $\beta_{x y}((\mathbf{m}-\mathbf{1})-S)>0$.

Now recall $[3, \S 6]$ that if $q$ is a fixed positive integer, then a $q$-lattice is a lattice $L$ of finite length with the property that every interval $[x, y]$ of $L$ for which $y$ is the join of atoms of $[x, y]$ is isomorphic to the lattice of subspaces of a projective geometry of degree $q$ (or to a Boolean algebra if $q=1$ ). Such a lattice is necessarily upper-semimodular [3, pp. 213-214] and hence possesses an admissible labeling. A $q$-lattice, however, need not be supersolvable, so the next proposition is strictly stronger than the corresponding Lemma 6.4 of [3]. For instance, let $L^{\prime}$ be the lattice of subgroups of a finite abelian $p$-group of type $(3,3)$. Let $L$ be $L^{\prime}$ truncated above rank 3 , i.e., identify all elements of $L^{\prime}$ of rank at least 4 . Then $L$ is a $p$-lattice but is not supersolvable.

4.6. PROPOSITION. (Replaces [3, Lemma 6.4]). Let $(L, \omega)$ be an admissible $q$-lattice of rank $n$. Let $S \subseteq \mathbf{n}-1$, with $(\mathbf{n}-1)-S=\left\{j_{1}, j_{2}, \ldots, j_{t-1}\right\}_{<}$. Also let $j_{0}=0$, $j_{t}=n$. Define $N(S)$ to be the number of maximal chains $K$ of $L$ satisfying $D\left(\pi_{K}\right) \supseteq S$, where $D\left(\pi_{K}\right)$ is the descent set of the $J-H$ sequence $\pi_{K}$. Then $N(S)=q^{k} M$, where

$$
k=\sum_{n=1}^{t}\left(\begin{array}{c}
j_{r}-j_{r-1} \\
2
\end{array}\right)
$$

and where $M$ is the number of Loewy chains

such that $\varrho\left(y_{i}\right)=j_{i}, 0 \leq i \leq t$.

$$
\hat{0}=y_{0}<y_{1}<\cdots<y_{t}=\hat{1}
$$


Since Proposition 4.6 is not a strict analogue of [3, Lemma 6.4], we shall give a proof.

Proof. If $K$ is a maximal chain of $L$ such that $D\left(\pi_{K}\right) \supseteq S$, then by Proposition 4.4 the subchain $C$ of $K$ consisting of all $x \in K$ such that $\varrho(x)=j_{i}(0 \leq i \leq t)$ is a Loewy chain. Hence it suffices to prove that if we have a Loewy chain (6) with $\varrho\left(y_{i}\right)=j_{i}$, then the number of refinements of $C$ to a maximal chain $K$ satisfying $D\left(\pi_{K}\right) \supseteq S$ is equal to $q^{k}$, where $k$ is given by (5).

Assume we have such a Loewy chain $C$. Since $L$ is a $q$-lattice, each interval $\left[y_{r-1}, y_{r}\right](1 \leq r \leq t)$ is a projective geometry of degree $q$ (or a Boolean algebra if $q=1)$. Hence $\mu\left(y_{r-1}, y_{r}\right)=(-1)^{b} q^{k_{r}}$, where $b=j_{r}-j_{r-1}$ and $k_{r}=\left(\begin{array}{c}j_{r}-j_{r-1} \\ 2\end{array}\right)$. Now by Corollary 3.3 the number of maximal chains $y_{r-1}=z_{0}<z_{1}<\cdots<z_{b}=y_{r}$ of the interval $\left[y_{r-1}, y_{r}\right]$ such that

$$
\gamma\left(z_{0}, z_{1}\right)>\gamma\left(z_{1}, z_{2}\right)>\cdots>\gamma\left(z_{b-1}, z_{b}\right)
$$

is just $(-1)^{b} \mu\left(y_{r-1}, y_{r}\right)=q^{k_{r}}$. Hence the total number of refinements of $C$ to a maximal chain $K$ satisfying $D\left(\pi_{K}\right) \supseteq S$ is equal to $q^{k_{1}} q^{k_{2}} \ldots q^{k_{t}}=q^{k}$, and the proof follows.

4.7. COROLLARY. (Generalizes [3, Corollary 6.5]). Let L be a q-lattice of rank $n$, and let $S \subseteq \mathbf{n - 1}$, with $(\mathbf{n - 1})-S=\left\{j_{1}, j_{2}, \ldots, j_{t-1}\right\}<$ and $j_{0}=0, j_{t}=n$. Then $\beta(S)$ is divisible by $q^{k}$, where $k$ is given by (6).

The derivation of Corollary 4.7 from Proposition 4.6 is not quite as trivial as the derivation of [3, Corollary 6.5] from [3, Lemma 6.4], so we shall give a proof.

Proof. Fix an admissible labeling $\omega$ of $L$. By Theorem $3.1, \beta(S)$ is equal to the number of maximal chains $K$ of $L$ satisfying $D\left(\pi_{K}\right)=S$. Hence if $N(S)$ is defined as in Proposition 4.6, we have

so

$$
N(S)=\sum_{T \supseteq \underline{S} S} \beta(T)
$$

$$
\beta(S)=\sum_{T \supset S}(-1)^{|T-S|} N(T)
$$

Suppose we have $\mathbf{n}-\mathbf{1} \supseteq T \supseteq S$ where $(\mathbf{n}-\mathbf{1})-T=\left\{i_{1}, i_{2}, \ldots, i_{s-1}\right\}<$ and $(\mathbf{n}-\mathbf{1})-S=$ $\left\{j_{1}, j_{2}, \ldots, j_{t-1}\right\}_{<}$, and $i_{0}=j_{0}=0, i_{s}=j_{t}=n$. An easy computation shows that

$$
\sum_{r=1}^{s}\left(\begin{array}{c}
i_{r}-i_{r-1} \\
2
\end{array}\right) \geq \sum_{r=1}^{t}\left(\begin{array}{c}
j_{r}-j_{r-1} \\
2
\end{array}\right) .
$$

It follows from Proposition 4.6 that each term $N(T)$ appearing in $(7)$ is divisible by $q^{k}$, so the proof follows. 
4.8. THEOREM. (Generalizes [3, Theorem 6.6]). Let $L$ be a q-lattice of rank $n$, and let $S \subseteq \mathbf{n}-1$ with $|S|=s$. Then $\beta(S)$ is divisible by $q^{Q(n, s)}$, where

$$
Q(n, s)=\frac{1}{2}\left[\frac{n}{n-s}\right]\left(n+s-(n-s)\left[\frac{n}{n-s}\right]\right)
$$

(brackets denote the integer part). This result is best possible in the sense that given $n$ and $0 \leq s \leq n-1$, there exists a q-lattice (which can even be chosen to be modular) of rank $n$ and $a$ set $S \subseteq \mathrm{n}-1$ of cardinality such that $\beta(S)=q^{Q(n, s)}$ (see $\left.[3, \mathrm{p} .216]\right)$.

\section{The broken circuit theorem}

In this section we shall point out the connection between our work and the socalled 'broken circuit theorem' of G.-C. Rota [2, Prop. 1, p. 358], which generalizes to arbitrary finite geometric lattices a result of Whitney on graphs. The reader should be warned that [2, Prop. 1, p. 358] is false when $k>1$. However, the proof is valid when $k=1$, and this is the case which will concern us here.

We proceed to describe the broken circuit theorem. Let $L$ be a finite geometric lattice of rank $n$, and let $a_{1}, a_{2}, \ldots, a_{t}$ be an ordering of the atoms $A$ of $L$. A subset $C$ of $A$ is called a circuit if the rank of the join of the elements of $C$ is $|C|-1$, while the rank of the join of the elements of any proper subset $C^{\prime}$ of $C$ is $\left|C^{\prime}\right|$. A subset $B=\left\{a_{i_{1}}, a_{i_{2}}, \ldots, a_{i_{j}}\right\}$ of $A$ is called a broken circuit if there exists an atom $a_{m}$ such that $m>i_{r}$ for $r=1,2, \ldots, j$, and such that $B \cup\left\{a_{m}\right\}$ is a circuit. Note that the notion of a circuit depends only on $L$, while that of a broken circuit also depends on the ordering chosen for the elements of $A$.

BROKEN CIRCUIT THEOREM (G.-C. Rota). Let $L$ be a finite geometric lattice of rank $n$ with an ordering $a_{1}, a_{2}, \ldots, a_{t}$ of the atoms of L. Let $\mu$ be the Möbius function of $L$. Then $(-1)^{n} \mu(\hat{0}, \hat{1})$ is equal to the number of sets of $n$ atoms of $L$ not containing any broken circuit.

Given an ordering $a_{1}, a_{2}, \ldots, a_{t}$ of the atoms of a finite geometric lattice $L$ of rank $n$, define a labeling $\omega$ of $L$ by $\omega\left(a_{i}\right)=t-i+1$, so $i<j$ implies $\omega\left(a_{i}\right)>\omega$. By Proposition 2.2, $(L, \omega)$ is an admissible lattice. Let $\hat{0}=x_{0}<x_{1}<\cdots<x_{n}=\hat{1}$ be a maximal chain $K$ in $L$ satisfying

$$
\gamma\left(x_{0}, x_{1}\right)>\gamma\left(x_{1}, x_{2}\right)>\cdots>\gamma\left(x_{n-1}, x_{n}\right) \text {. }
$$

We know by Corollary 3.3 that the number of such maximal chains $K$ is $(-1)^{n} \mu(\hat{0}, \hat{1})$. We would like to relate this fact to the Broken Circuit Theorem by constructing an explicit one-to-one correspondence $\lambda$ between maximal chains $K$ satisfying (8) and sets of $n$ atoms of $L$ containing no broken circuit. 
This correspondence $\lambda$ is defined as follows. Given a maximal chain $K$ satisfying (8), let $\lambda(K)$ be the set $\left\{b_{1}, b_{2}, \ldots, b_{n}\right\}$ of those $n$ atoms defined by $\omega\left(b_{j}\right)=\gamma\left(x_{j-1}, x_{j}\right)$.

5.1. PROPOSITION. The function $\lambda$ defines a one-to-one correspondence between maximal chains $K$ of $L$ satisfying (8), and sets of $n$ atoms of $L$ containing no broken circuit.

Proof. We first prove that $\lambda(K)$ contains no broken circuits. Suppose $B=\left\{b_{i_{1}}, b_{i_{2}}\right.$, $\left.\ldots, b_{i_{s}}\right\}$ is a broken circuit contained in $\lambda(K)$ with $i_{1}<i_{2}<\cdots<i_{s}$, i.e., $\omega\left(b_{i_{1}}\right)>\omega\left(b_{i_{2}}\right)$ $>\cdots>\omega\left(b_{i_{s}}\right)$. By definition of broken circuit, there exists an atom $a$ of $L$ such that $\omega\left(b_{i_{r}}\right)>\omega(a)$ for $r=1,2, \ldots, s$ and $B \cup\{a\}$ is a circuit. By definition of the $b_{i}$ 's and $\gamma, x_{i_{s-1}} \vee b_{i_{s}}=x_{i_{s}}$ and $b_{i_{r}} \leq x_{i_{s-1}}$ for $r=1,2, \ldots, s-1$. Hence since $B \cup\{a\}$ is a circuit, $x_{i_{s-1}} \vee a=x_{i_{s}}$. By definition of $\gamma$, this means $\omega\left(b_{i_{s}}\right)<\omega(a)$, a contradiction. Hence $\lambda(K)$ contains no broken circuit.

Now let $B=\left\{b_{1}, b_{2}, \ldots, b_{n}\right\}$ be a set of $n$ atoms containing no broken circuit, with $\omega\left(b_{1}\right)>\omega\left(b_{2}\right)>\cdots>\omega\left(b_{n}\right)$. Recall that a basis of $L$ is a set of $n$ atoms $c_{1}, c_{2}, \ldots, c_{n}$ of $L$ such that $\varrho\left(c_{1} \vee c_{2} \vee \cdots \vee c_{n}\right)=n$. Equivalently, a basis is a set of $n$ atoms containing no circuit. Now note that $B$ is a basis, since if it contained a circuit it would contain a broken circuit. If $\lambda(K)=B$, then $K$ must be given by $x_{j}=b_{1} \vee b_{2} \vee \cdots \vee b_{j}$, so $\lambda$ is injective. It remains to prove that these $x_{j}$ 's satisfy $\gamma\left(x_{j-1}, x_{j}\right)=\omega\left(b_{j}\right)$, which shows $\lambda$ is surjective. By definition of the $x_{i}$ 's, $x_{j-1} \vee b_{j}=x_{j}$. Suppose $a$ is an atom such that $x_{j-1} \vee a=x_{j}$ and $\omega(a)<\omega\left(b_{j}\right)$. Thus the set $\left\{b_{1}, b_{2}, \ldots, b_{j}, a\right\}$ contains a circuit $C$. Moreover, $a \in C$ since the $b_{i}$ 's are independent. Since $\omega\left(b_{1}\right)>\omega\left(b_{2}\right)>\cdots>\omega\left(b_{j}\right)$ and $\omega\left(b_{j}\right)>\omega(a), \omega(a)<\omega\left(b_{i}\right)$ for $1 \leq i \leq j$. Hence $C-\{a\}$ is a broken circuit, a contradiction. This completes the proof.

\section{REFERENCES}

[1] Garrett Birkhoff, Lattice Theory, 3rd Ed., American Mathematical Society, Providence, Rhode Island, 1967.

[2] .G.-C. Rota, On the foundations of combinatorial theory: I: Theory of Möbius functions, Z. Wahrscheinlichkeitstheorie, 2 (1964), 340-368.

[3] R. Stanley, Supersolvable lattices, Algebra Universalis, 2 (1972), 197-217.

University of California at Berkeley Berkeley, California

U.S.A. 